\title{
RETRODATAZIONI DA FUMETTI
}

\author{
Mario Piotti
}

Che il fumetto seriale fosse luogo di una, talora impacciata, conservazione linguistica è osservazione che giunge da più specole. Già l'analisi pionieristica di Nencioni metteva in luce questo aspetto, segnalando, in negativo, nei fumetti degli anni sessanta del Novecento «il prevalere della lingua comune nella sua maggiore passività [...] e nella sua elementarità sintattica; [...] e diffuso, piuttosto, un ibridismo interno tra mal fusi livelli della stessa lingua» ${ }^{2}$. Qualche anno dopo, accentuando valutativamente il prevalente descrittivismo nencioniano, al quale per altro dichiaratamente si rifaceva, Emanuela Cresti, in uno studio che metteva a confronto la lingua della satira a fumetti con quella del fumetto tradizionale, confermava la natura di quest'ultima come una variante povera dell'italiano standard e la passività, pur se segnalava una diminuzione delle tendenze puristiche ${ }^{3}$.

Da parte sua Ivano Paccagnella, studiando la lingua del più longevo tra i fumetti italiani, Tex, e mettendone in luce i tratti tradizionali di una lingua orientata sul piano alto, notava che «la vicenda linguistica di Tex-Bonelli è quella di un parlante medio-colto, formatosi nel primo ventennio del secolo [del '900], con una scolarizzazione che risente del manzonismo sedimentato della scuola e nella narrativa di consumo» ${ }^{4}$.

Non di necessità, tuttavia, la constatazione della resistenza di una lingua tradizionale si accompagnava alla valutazione negativa. Così Fabio Rossi descriveva la serie dei tratti tradizionali (resistenza di egli, di loro come dativo plurale; tenuta del congiuntivo, del condizionale, del futuro, del passato remoto); ma sottolineava anche, almeno nei fumetti della Disney, la capacità di «coniugare agilità sintattica e formalità, rispetto della norma e impiego ironico della letterarietà, specificità lessicale e composte aperture al parlato» ${ }^{5}$. Ai fumetti Disney era dedicato anche il poderoso studio di Daniela Pietrini che, pur riconoscendo presenze tradizionali e anche letterarie, ne sottolineava l'atteggiamento linguistico tutt'altro che passivo e il ruolo importante svolto nel rinnovamento dell'italiano contemporaneo ${ }^{6}$

Pur nella differenza di valutazione, il riconoscimento del dato tradizionale e letterario è dunque una costante. E se si respinge, almeno in parte, la semplice spiegazione di presenze

\footnotetext{
${ }^{1}$ Università degli Studi di Milano.

${ }^{2}$ Nencioni 1976, p. 11.

${ }^{3}$ Cfr. Cresti 1992. pp. 111-116.

${ }^{4}$ Paccagnella 2002, p. 620.

${ }^{5}$ Rossi 2010.

${ }^{6}$ Cfr. Pietrini 2007. Il rimando è necessariamente a tutto il volume, ma per le considerazioni qui riprese si vedano in particolare le pp. 393-401.
} 
inerziali, la motivazione potrà forse essere recuperata nelle aspettative dei lettori, pur se di differenti fasce anagrafiche. Come ha scritto Vittorio Spinazzola, anche il destinatario dei fumetti legge formalisticamente, indipendentemente dalla sua cultura, e nella letteratura disegnata cerca l'applicazione di «canoni retorici e stilistici adeguati alla sua competenza»?

Se anche i dati della tradizione si accampano a tutti i livelli, certamente colpisce la loro presenza nel lessico: la voce o la locuzione schiettamente e riconoscibilmente letteraria da un lato può essere riconducibile a una semplice ricerca di sostenutezza di registro; altre volte invece, e specialmente nei fumetti Disney e più in generale in quelli destinati alle fasce anagraficamente più giovani, risponde a un intento ludico-espressivo ${ }^{8}$. E all'aspetto espressivo, se non a quello ludico, contribuisce una serie di elementi lessicali di più defilata letterarietà ma, se non sempre, appartenenti alla dimensione tradizionale, spesso riconducibili alla fraseologia: a essi il fumetto, anche quello per adulti, affida con frequenza la resa della colloquialità.

A questo secondo gruppo di presenze tradizionali sono dedicate le retrodatazioni che qui si presentano, ricavate attraverso il ricorso a GoogleLibri. Si tratta di voci e locuzioni, individuate nel corso di lavori dedicati, in particolare ma non solo, ai fumetti della casa editrice Bonelli ${ }^{9}$, che sono spesso legate ai fini espressivi e che collaborano a fare della lingua stessa una componente iconografica. Proprio la numerosità di locuzioni verbali nei fumetti suggerisce la presenza di un'espressività che sembra volersi cimentare non solo nel puro descrittivismo, ma che informa anche la natura delle azioni. Per alcune di queste voci non è tanto l'anagrafe a determinarne l'assegnazione alla tradizione, quanto la modalità compositiva, come ad esempio per le uniche due presenze non polirematiche mangiacrauti e scannagatti - che sono entrambe di attestazione solo ottocentesca, ma la cui modalità compositiva - verbo + sostantivo - è antichissima; mentre per molte delle locuzioni verbali si sono recuperate attestazioni anche cinque-secentesche. Va detto che tutte le voci retrodatate sono indicate dal GRADIT, se presenti, con la marca "comune", cioè sono generalmente note a chi abbia un livello di istruzione mediosuperiore; tuttavia nella maggior parte dei casi le motivazioni che ne hanno determinato la nascita, radicate nella società che ne vide la luce, non appaiono più centrali nella società contemporanea e, in alcuni coasi, si sono opacizzate: e ciò, per molte di esse, vale come conferma della tradizionalità, quantomeno dal punto di vista motivazionale ${ }^{10}$.

\section{AVERE IL DENTE AVVELENATO}

loc. v. 'provare risentimento, rancore'

DELIN: $1961 \rightarrow \mathbf{1 8 3 2}$

\footnotetext{
${ }^{7}$ Spinazzola 2001, pp. 395-396.

${ }^{8}$ Cfr. Pietrini 2007, pp. 100-108.

${ }^{9}$ Cfr., per il corpus di fumetti su cui si è lavorato, Piotti 2018 (dove un'altra manciata di retrodatazioni) e Piotti 2020, pp. 41-60. Sul lavoro di retrodatazione si veda, da ultimo, Maconi 2020.

${ }^{10}$ Prendo come modello per le schede seguenti, con minimo scostamento, quello proposto in Biffi, Paoli, Setti 2020; le voci segnalate con un asterisco indicano parole assenti o prive di datazione negli strumenti lessicografici consultati. Quasi tutte le voci sono corredate solo dall'esempio più antico rintracciato; in pochi casi mi è parso opportuno aggiungere al primo uno o due altri esempi.
} 
Dialoghi disposti per facilitare lo studio della lingua italiana scritti in francese da A. G. Collot tradotti da F. Mancinelli romano, Philadelphia, Carey \& Lea, 1832, p. 85: «Sembra che voi abbiate il dente avvelenato contro i medici».

AVERE LA BOCCA CUCITA

loc. v. 'non parlare per mantenere un segreto'

GDLI: $1758 \rightarrow 1663$

Giacinto Andrea Cicognini, La vita è un sogno, Bologna, Carl'Antonio Peri, All'Insegna dell'Angelo Custode, 1663, p. 28: «Rosaura. Taci indegno, Oh Dio. - Piccariglio. Non ho la bocca cucita. Vuò parlare».

\section{AVERE LA CODA DI PAGLIA}

loc. v. 'sentirsi in difetto o in colpa per qualcosa e pensare che gli altri vi alludano' GDLI, DELIN: $1846 \rightarrow 1605$

Alessandro Donzellini, Tempesta amorosa comedia, Venezia, Roberto Meglietti, 1605, p. 157: «Se tu hai la coda di paglia, io ho il solfinello»; Giulio Varrini, Scuola del volgo, cioè Scielta de" più leggiadri, e spiritosi, detti, aforismi, e proverbi [...], Venezia, Francesco Roisi, 1642, p. 129: "Quel che hà la coda di paglia, hà paura del fuoco».

\section{AVERE UN DIAVOLO PER CAPELLO}

loc. v. 'avere molte preoccupazioni, problemi; essere molto nervoso'

DELIN: $1875 \rightarrow 1808$

Filippo Pananti, Il poeta di teatro, 1808: «L'Imbratta aveva un diavol per capello, Faceva salti come un capriolo» ${ }^{11}$.

\section{CERCARE UN AGO IN UN PAGLIAIO*}

loc. v. 'cercare una cosa piccola in uno spazio enorme'; 'tentare una ricerca impossibile' Datazione: 1844

Cesare Cantù, Milano e il suo territorio, t. II, Milano, Pirola, 1844, p. 196: «Se si conosca il nome del notaro, facilmente può trovarsi qualunque rogito; ma se s'ignori, è un cercare l'ago in un pagliaio».

${ }^{11}$ Cito dalla terza edizione italiana, Firenze, Piatti, 1824: 53. L'opera uscì in prima edizione a Londra nel 1808. 


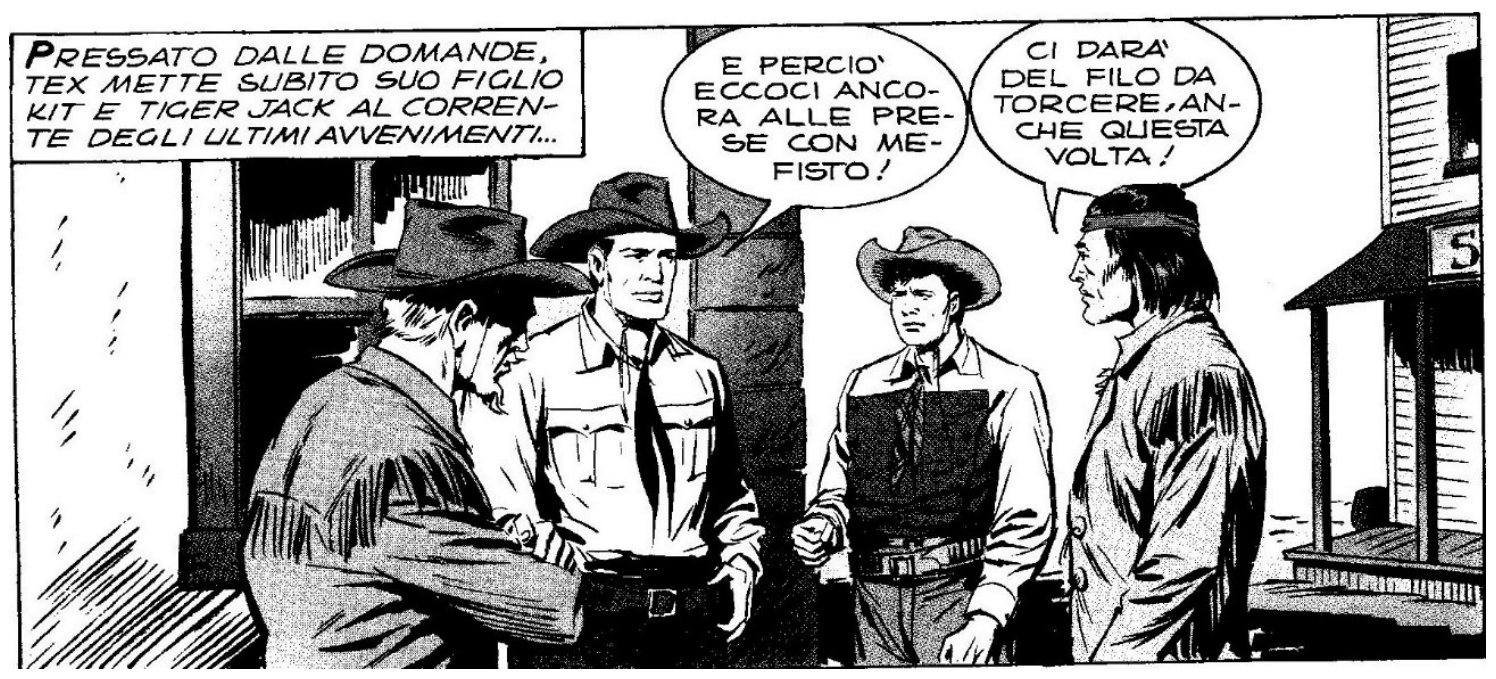

Texn. $93-1968$

DARE FILO DA TORCERE

loc. v. 'causare difficoltà; rendere la vita difficile'

GDLI, DELIN: $1898 \rightarrow \mathbf{1 7 9 8}$

Francesco Beccatini, Vita e fasti di Caterina II imperatrice ed autocratrice di tutte le Russia ec. ec., Lugano [Venezia], Antonio Zatta, 1798, t. IV, p. 56: «Il Marchese di Pugatschef mi ha dato tanto filo da torcere, contro ogni mia espettazione, che per più di otto settimane non ho dovuto occuparmi in altro che in quest'affare».

\section{DRIZZARE LE ANTENNE*}

loc. v. 'drizzare le orecchie; prestare grande attenzione'

Datazione: 1979

L'Europeo, 35, 1979, p. 68: «E se per ora consumeranno pochi cosmetici, potranno fabbricarne in grandi quantità, e a buon mercato: ciò che ha già fatto drizzare le antenne a qualche gigante dell'industria cosmetica francese».

FARE FUOCO E FIAMME

loc. v. 'agitarsi e strepitare'

DELIN: $1869 \rightarrow 1771$

François Alberti, Nouveau dictionnaire français-italien, Marseille, Jean Mossy, 1771, s.v. flamme: «Jeter feu \& flamme, pour dire, parler en homme transporté de colère. Gettare, e far fuoco e fiamma».

GIÙ DI CORDA*

loc. agg. inv. 'giù di morale'

Datazione: 1878

Bollettino del Club Alpino Italiano, vol. 12, 1878, p. 175: «Intanto il morale è giù di corda».

IMBRATTACARTE

s.m. e f.: 'scrittore di nessun valore';

GDLI, DELIN e GRADIT: $1563 \rightarrow 1552$ 
Anton Francesco Doni, Fiori della Zucca, Venezia, Francesco Marcolini, 1552, p. 17: «Però bisogna che noi altri imbratta carte facciamo opere così per i dottori da vero come da beffe».

\section{LAVARE I PANNI SPORCHI IN FAMIGLIA}

loc. v. 'risolvere le questioni delicate o spiacevoli all'interno dell'ambiente in cui sono sorte, senza renderle pubbliche'

GDLI: $1889 \rightarrow \mathbf{1 8 2 0}$

Massime e pensieri del prigioniere di Sant'Elena. Manoscritto trovato nelle carte di Las-Casas, tradotto dal francese da Felice Miola, Napoli, Tipografia di Porcelli, 1820, p. 98: «È in famiglia che bisogna lavare i panni sporchi».

\section{MANGIACRAUTI}

s.m e f. 'tedesco'

GDLI e GRADIT: sec. XX $\rightarrow \mathbf{1 8 8 2}$

Cristoforo Pasqualigo, Raccolta di proverbi veneti, terza edirione accresciuta dei proverbi delle Alpi Carniche, del Trentino e dei tedeschi dei Sette Comuni vicentini, Treviso, Zoppelli, 1882, di Cristoforo Pasqualigo, p. 263: «magnacrauti da Roncegno».

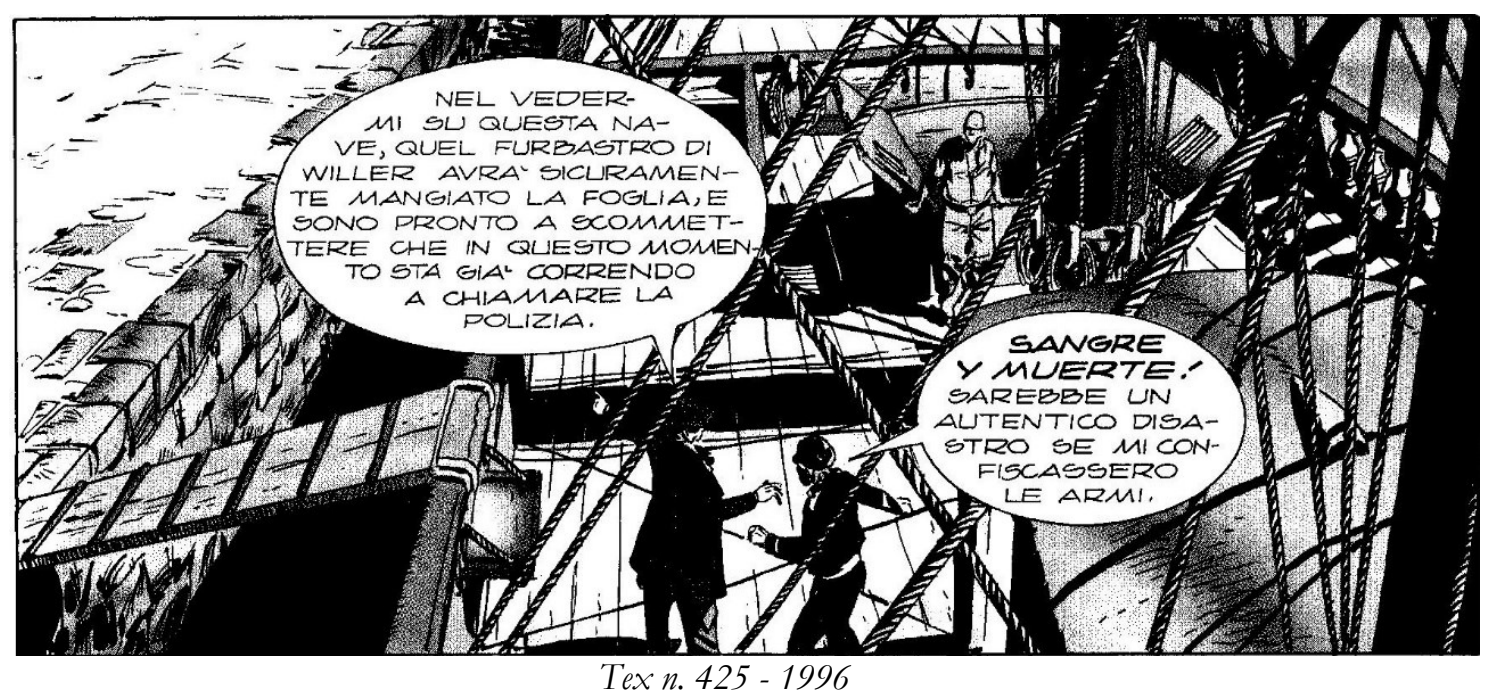

MANGIARE LA FOGLIA

loc. v. 'intuire la verità, accorgersi di un inganno'

DELIN: $1869 \rightarrow \mathbf{1 8 3 9}$

Tribunale supremo della Sagra Consulta - Turno speciale - Ravenna Omicidio insidioso in odio, e per inspirito di parte nella persona di Luigi Cottignola di Ravenna [...], Roma, Stamperia della reverenda Camera Apostolica, 1839, p. 30: «Quella, che non si mettesse altramente le mani alla faccia al trasentito colpo; ma che avendo mangiata la foglia, come a dire, essersi accorto, che si era tirato per uccidere il Maestro a di lui lato, guardasse bene tosto all'angolo della discontro Sartoria Pascoli».

\section{METTERE I BASTONI TRA LE RUOTE}

loc. v. 'ostacolare'

GDLI e DELIN: $1894 \rightarrow \mathbf{1 8 4 8}$ 
Raccolta per ordine cronologico di tutti gli atti, decreti, nomine ecc. del Governo provvisorio di Venezia, Venezia, Andreola Tipografo del Governo provvisorio, 1848, p. 413: «senza sospetto che i principi gli mettano il bastone fra le ruote del carro».

\section{METTERE UNA PIETRA SOPRA}

loc.v. 'rinunciare definitivamente a qualcosa'

GDLI: $1855 \rightarrow 1794$

Antonio Eximeno, Lo spirito del Machiavelli, Cesena, eredi Biasini all'Insegna di Pallade, 1794, p. 84: «A' Santi costa moltissimo, che intorno a' lor peccatuzzi (e ne commettono alla giornata almeno sette) si metta una pietra sopra; ma perchè a' letterati (che appunto perchè lo sono, poco danno da fare alla Congregazione de' Riti) perchè, dico, hannosi da inorpellare, e peccatuzzi, e peccati?».

\section{NON CAVARE UN RAGNO DAL BUCO}

loc. v. 'non riuscire a ottenere nulla relativamente allo scopo prefisso'

GDLI: $1827 \rightarrow \mathbf{1 7 2 9}$

Vocabolario degli Accademici della Crusca, quarta impressione, vol. I, Firenze, Manni, 1729, s.v. cavare: «Non sapere, o non potere cavare un ragno d'un buco, dicesi proverbialm. di uomo dappoco, di chi abbia pochissima abilità. Lat. haerere in re facilì. Giampietro Bergantini, Della volgare locurione illustrata, ampliata, facilitata, Venezia, Lazzaroni, 1740, p. 750: «Chi per la sua dappocaggine nè anche le cose facilissime sappia fare. E' non sa cavar fuora un ragno da un buco». Annibale Antonini, Dictionnaire italien latin et françois [...], nouvelle édition, T. I, Lyon, chez les Freres Duplain, 1760, p. 138: «Non sapere, o non potere cavare un ragno d'un buco, dicesi d'Uomo dappoco, e di Pochissima abilità».

NON VALERE LA CANDELA

loc. v. 'non valere la pena'

ArchiDATA: $1909 \rightarrow \mathbf{1 8 2 4}$

Nuovo Giornale de' Letterati d'Italia, tomo VI, 1773, p. 240: «grande essere stata la fatica: generosa l'Inglese ricompensa: ma il giuoco non valere la candela»; Giornale di Scienze Lettere ed Arti per la Sicilia, t. V, a. II, 1824, p. 195: «ben si potrebbe dire con Montaigne, che il gioco non varrebbe la candela».

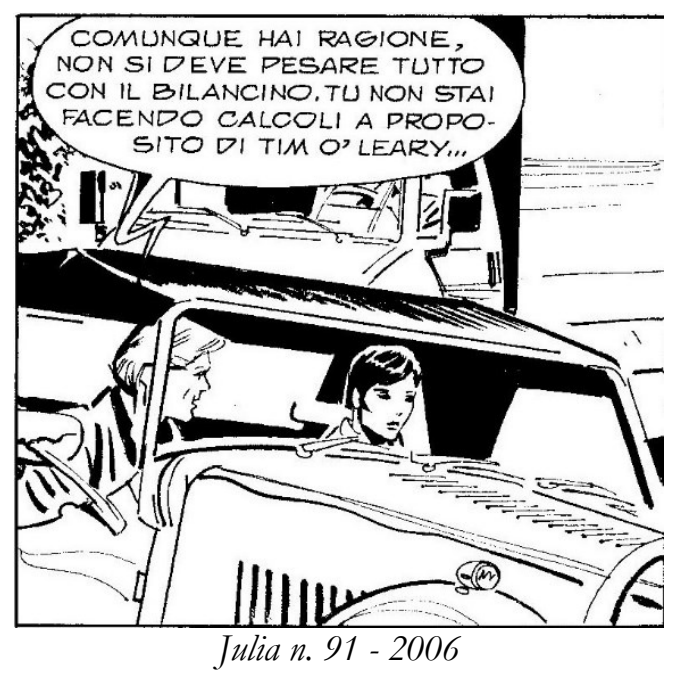


PESARE COL BILANCINO*

loc. v. 'esaminare con estrema scrupolosità

Datazione: 1650

Giovanni Andrea Alberti, Enneade Panegirica detta a S. Francesco Saverio, Bologna, Carlo Zenero, 1650, p. 26: «pesale [le colpe] col bilancino de' Santi, ne' quali co' prezzi eterni son valutati i momenti: tanto gli sono grievi, che sotto l'incarco di esse quasi di monti, il vedi qual altro Publicano incurvato».

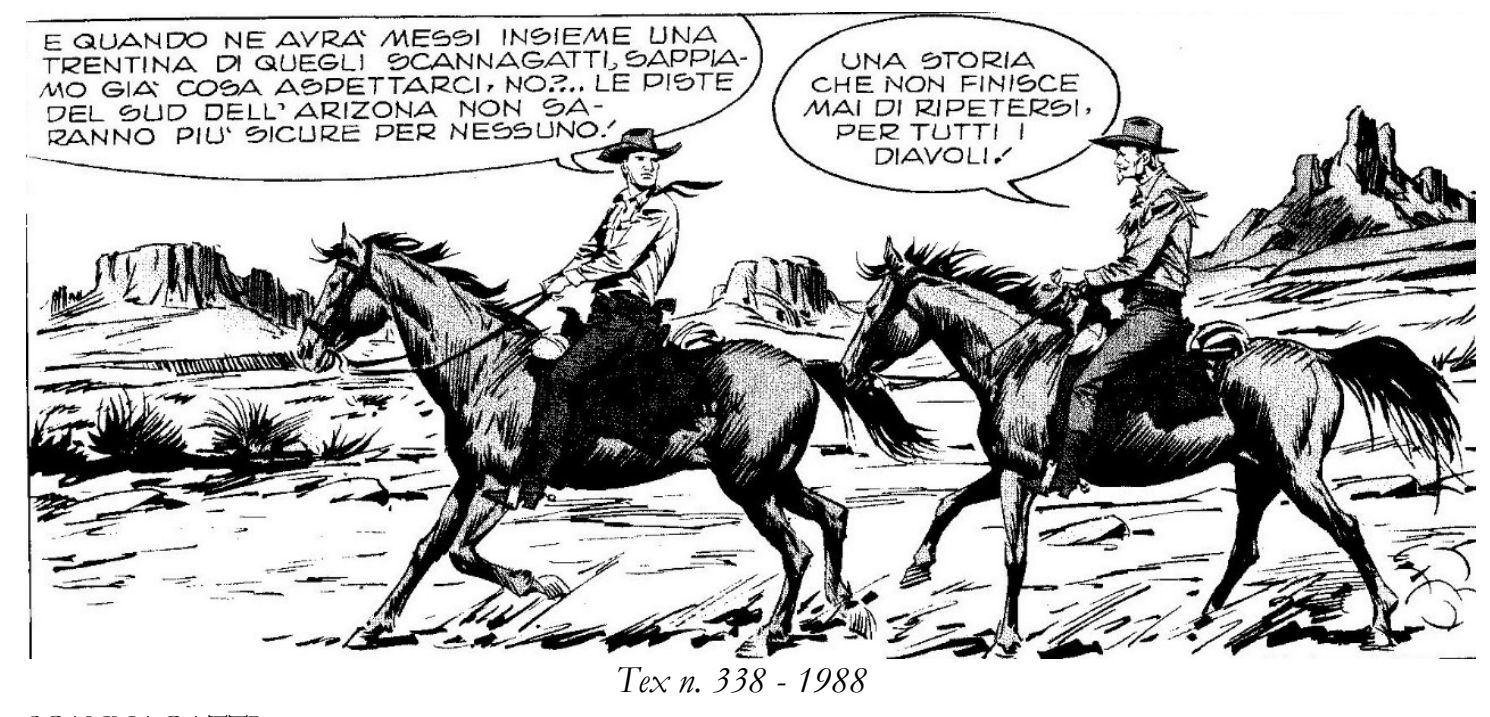

SCANNAGATTI

s.m e f. 'delinquente di scarsa abilità'

Datazione: 1815

Documentato dapprima nella lessicografia dialettale piemontese (scanagat): Casimiro Zalli, Disionari piemontèis, italian, latin e fransèis, vol. II, Peder Barbiè, Carmagnola, 1815, s.v.: «Scanagat, mascalzone, povero in canna, uomo vile, guidone, piccaro, pitocco, pelapiedi, scalzagatto, scalzacane, miserrimus, furcifer, hono trioboli, vile caput, gueux, poiloux, gredin, pied-poudreux, miserable, galefretier, pékin»; Michele Ponza, Vocabolario piemonteseitaliano, vol. 3, Torino, Stamperia reale, 1833, s.v.: «SCANAGAT, $n$. mascalzone, faccimale, malbigatto, dappoco, pitocco, pelapiedi, scalzagatto, scalzacane». Ma è anche nel romanzo dello scrittore toscano, e toscanista, Giovanni Rosini, Luisa Strozzi: storia del secolo XVI, T. III, Pisa, Tipografia di N. Capurro e Comp., 1832. p. 134: «L'insolenza di questi scannagatti è arrivata al punto che non si può sopportare». La fortuna fumettistica della voce sarà forse anche legata al maestro Antonio Scannagatti, interpretato da Totò. Voce non documentata dai dizionari. 


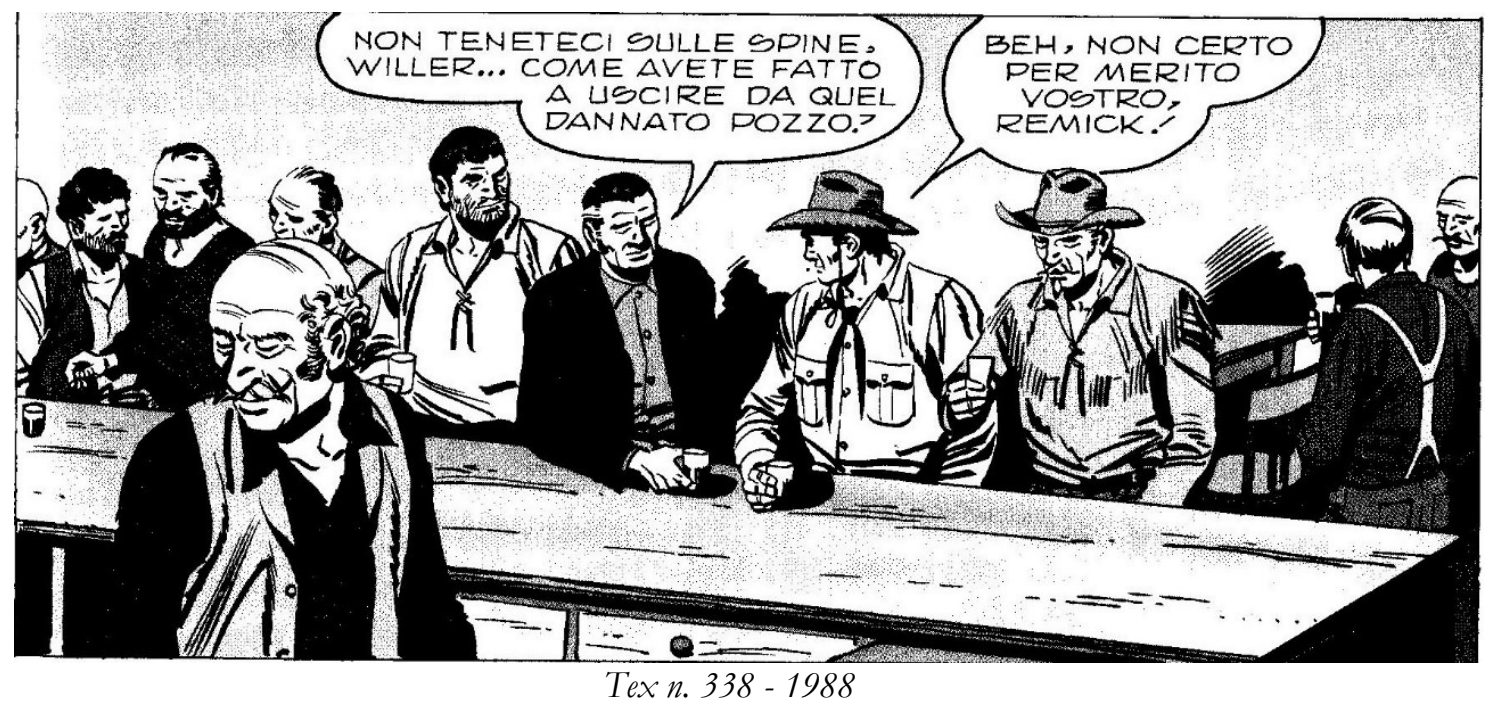

TENERE SULLE SPINE

GDLI: $1946 \rightarrow \mathbf{1 7 6 0}$

loc. v. 'tenere in uno stato d'ansia, di preoccupazione'

Giovanni Castiglioni, Prefarione a Alexander Pope, Saggio sull'uomo [...] tradotto dall'inglese dal sig. Gio. Castiglioni, Berna, Wagner, 1760, p. XXII: «quei dotti riservati e sentenziosi che tengono gli uditori sulle spine e stuccano mentre si fanno ammirare».

\section{RIFERIMENTI BIBLIOGRAFICI}

ArchiDATA, Archivio datazioni lessicali, https://www.archidata.info/

Biffi M., Paoli M., Setti R. (2020), "(Retro)datazioni dalle risposte del servizio di consulenza della Crusca (2017-2019)", in Maconi L. (a cura di), pp. 209-238.

Cresti E. (1992), "La lingua della satira a fumetti", in A.A.V.V., Gli italiani scritti, Incontri del Centro di studi di grammatica italiana, Firenze 22-23 maggio 1987, pp. 111-152.

GDLI: Grande dizionario della lingua italiana, fondato da S. Battaglia, 21 voll. e 2 supplementi, Utet, Torino, 1961-2008.

GRADIT: Grande dizionario italiano dell'uso, ideato e diretto da T. De Mauro, 8 voll., Utet, Torino, 1999-2007.

DELIN: Manlio Cortelazzo e Paolo Zolli Dirionario etimologico della lingua italiana, seconda edizione, Zanichelli, Bologna, 1999.

Maconi L. (2020) (a cura di), Laboratorio di ArchiD ATA 2020. Retrodatarioni lessicali: storia di cose e di parole, Accademia della Crusca, Firenze. 
Nencioni G. (1977), "Prefazione" a Leonardo Becciu, Il fumetto in Italia, Sansoni, Firenze, pp. 7-14.

Paccagnella I. (2002), “"Valgame Dios, pards!». Così parlò Tex Willer”, in Beccaria G.L., Marello C. (a cura di), La parola al testo. Scritti per Bice Mortara Garavelli, 2 voll., Edizioni dell'Orso, Alessandria, pp. 605-620.

Pietrini D. (2007), Parola di papero. Storia e tecniche della lingua dei fumetti Disney, Cesati, Firenze.

Piotti M. (2018), "I giornali e i fumetti per il VoDIM - Vocabolario Dinamico dell'Italiano Moderno", in «Lingue e Culture dei Media», 2, 2, pp. 62-90.

Piotti M. (2020), Tra fumetti e Tv. Studi sulla lingua dei media, Milano, Ledizioni.

Rossi F. (2010), "fumetti, linguaggio dei", in Enciclopedia dellitaliano, Treccani https://www.treccani.it/enciclopedia/linguaggio-dei-fumetti (Enciclopediadell'Italiano)/

Spinazzola V. (2001), La modernità letteraria, Il Saggiatore - Fondazione Mondadori, Milano. 